Malaquias Batista Filho 1

Luiz Oscar Cardoso Ferreira 2

\title{
Prevenção e tratamento da anemia nutricional ferropriva: novos enfoques e perspectivas
}

\author{
Prevention and treatment of iron-deficiency \\ anemia: new focuses and perspectives
}

1 Instituto Materno Infantil de Pernambuco

Rua dos Coel hos 300, Recife, PE 50070-550, Brasil.

2 Faculdade de Ciências Médicas, Universidade de Pernambuco.

Rua Arnóbio Marques s/no, Recife, PE 50100-130, Brasil.
A bstract The authors briefly describe the epidemi ology of anemia, the goals established by the United Nations to combat the problem worldwide, and the difficulties encountered in applying proven effective strategies in public health services to prevent and cure anemia in pregnant women and children, both of which are prime high-risk groups. They analyze recent research aimed at four objectives related to prevention and cure: improving treatment efficacy; increasing effectiveness; reducing costs; and decreasing unwanted si de effects. The study indicates that ongoing research could improve prospects for treatment through public health services. Key words Anemia; Iron; Diet; Nutrition; Public Health

Resumo Os autores descrevem sumariamente o quadro epidemiológi co das anemias, as propostas programáti cas estabel eci das pelas Nações Uni das para a reversão do problema em escala internacional e as dificuldades atuais para a apl icação de estratégi as de reconheci da efeti vidade na área da saúde para a prevenção e cura das anemias nos segmentos populacionais mais expostos, as crianças e as gestantes. Analisam o estági o atual das pesqui sas dirigi das para quatro objetivos simultâneos: aumento da efi cácia e efetividade, redução de custos e de efeitos colaterais dos esquemas medi camentosos de prevenção e tratamento. Consi deram que as pesquisas em curso podem melhorar radicalmente as perspectivas de uma intervenção bem su cedi da no enfrentamento do problema através do setor saúde.

Palavras-chave Anemia; Ferro; Dieta; Nutrição; Saúde Pública 


\section{Introdução}

As anemias nutricionais constituem o maior problema nutricional da atualidade, estimando-se que 2,150 bilhões de pessoas, quase $40 \%$ da população mundial, apresentam carência de ferro ou níveis baixos de hemoglobina (Viteri et al., 1993). Mesmo na Europa, 27 milhões de habitantes estariam nesta condição, estabelecendo uma situação de risco que inclui até famílias de renda média e elevada, especialmente gestantes e crianças menores de dois anos de idade (Arruda, 1990; Rodrigues, 1989). É consenso entre os estudiosos que a deficiência de ferro é o principal responsável pelas anemias nutricionais (OMS, 1975; Viteri et al., 1993; Suharno et al., 1993). Em sete países latino-americanos, a prevalência da anemia em mulheres na idade reprodutiva foi de $21 \%$ das não grávi das e de $48 \%$ das gestantes (Ebrahim, 1983).

Estudos sobre anemia em crianças e gestantes brasileiras, grupos reconhecidamente como de maior risco, mostram sempre uma prevalência elevada. O ICNND encontrou em 1963, no Nordeste do Brasil, 22,3\% e 20,2\% de anemia entre menores de cinco e de 15 anos de idade respectivamente (ICNND,1965). Doze anos depois, em quatro localidades do Estado de Pernambuco, Salzano et al. (1975) detectaram uma prevalência de 35,1\% de anêmicos em menores de seis anos de idade. Em São Paulo, Szarfarc (1972) asssinalou anemia em 52,1\% das parturientes e em $21 \%$ dos recém-nascidos. Em outra investigação, o mesmo autor (Szarfarc, 1983), estudando gestantes também no Estado de São Paulo, encontrou uma ocorrência de 35,1\% de anêmicas, enquanto Rodrigues (1989), no mesmo estado e grupo etário, encontrou uma prevalência de $29,2 \%$. Mais recentemente, Torres et al. (1993) descreveram, no grupo etário de seis a 23 meses, 72,2\% de anêmicos em Ibiúna, São Paulo. Em Pernambuco, na cidade de Recife, Arruda (1990), estudando gestantes e parturientes, encontrou anemia em $30,3 \%$ e $38,4 \%$ de cada grupo, respectivamente. As implicações das anemias parecem bem estabel ecidas na redução da atividade física, do rendimento do aprendizado e do comprometimento dos sistemas de defesa imunológica, facultando a ocorrência e/ ou o agravamento de doenças infecciosas. (Arruda, 1990; Mahomed \& Hyten, 1993; Viteri et al., 1993).

O reconhecimento desta situação tem sensibilizado estudiosos dos problemas de saúde e nutrição, até então preocupados com nosologias mais ostensivas ou de efeitos mais notáveis, como a desnutrição energético-protéico
(DEP), a deficiência de iodo e a hipovitaminose A. Mais recentemente, passou-se a valorizar a ocorrência das anemias nutricionais como problema de importância epidemiológica relevante. Até então, devido à baixa eficácia do tratamento medicamentoso, o tema fora negligenciado, pois, pela pobreza de sintomas e elevada incidência de efeitos colaterias indesejáveis, houve pouca aderência às recomendações terapêuticas. Este artigo trata de levantar a discussão sobre o enfrentamento do problema a partir de concepções mais recentes, utilizando baixas doses de ferro elementar, o que poderia contornar os problemas historicamente acumulados da terapia medicamentosa e melhorar consideravel mente o sucesso de intervenções específicas do setor saúde.

\section{Compromissos programáticos}

Em 1990, a Reunião de Cúpula de Nova York estabeleceu, pela primeira vez em instância internacional, o combate à anemia nutricional ferropriva como uma das prioridades de nutrição na área de saúde (UNICEF, 1990). Definiuse, na oportunidade, a meta de reduzir em um terço a prevalência das anemias em mulheres no período reprodutivo, enfatizando-se a gravidez como uma condição particularmente importante, tendo em vista as implicações das anemias no binômio mãe/ feto. Este posicionamento técnico e político valida, de certa forma, recomendações anteriores dos Comites de Especialistas da Organização Mundial, aconseIhando a administração sistemática de sais de ferro durante o pré-natal em todos os países, inclusive em áreas de baixa endemicidade (INACG, 1977).

A meta de controle da anemia nutricional ferropriva torna-se um tanto modesta em relação ao que se propõe para outros problemas, como é o caso do controle virtual do bócio e da deficiência de vitamina A e da redução em 50\% da prevalência da desnutrição moderada e grave, em todos os países do mundo, até o ano 2000 (UNICEF,1990).

$\mathrm{Na}$ Conferência Internacional sobre Nutrição, a Conferência de Roma, realizada em 1992, foram referendadas as metas da Reunião de Nova York, tendo sido enfatizada a necessidade de investigações sobre anemias. Em relação à criança, no entanto, o documento é omisso, mostrando possivelmente a grande dificuldade de se enfrentar o problema com o conhecimento atualmente disponível. 
Enfrentamento da anemia: alguns problemas

A prevenção e terapêutica contra a anemia nutricional ferropriva a partir da utilização de compostos de ferro é um dos mais antigos recursos de tratamento que, ainda hoje, considera-se válido.

O paradigma historicamente mais remoto dos modelos de prevenção e tratamento consistiria no emprego de um prego implantado em um limão, utilizando-se o suco da fruta no dia seguinte, constituindo uma engenhosa estratégia de uso simultâneo de ferro (a conhecida "ferrugem", que manchava de vermelho-escuro o limão) e vitamina $C$, até então desconhecida. É também desta fase a recomendação de se usar o sangue e vísceras dos animais na alimentação como meio de combater as anemias. Estas experiências precederam a utilização farmacológica do ferro em escala industrial, mediante diferentes combinações de compostos orgânicos e inorgânicos do metal.

As perspectivas profiláticas e terapêuticas aumentaram com a descoberta de outros princípios hematínicos (ácido fólico, vitamina B12, zinco e, mais recentemente, vitamina $A$ ), assim como com o papel exercido pelas substâncias facilitadoras (vitamina C, leite humano) ou inibidoras (fitatos, carbonatos, polifenóis, medicamentos alcalinizantes etc.) do aproveitamento biológico do ferro (OMS, 1975; Mahomed \& Hyten, 1993).

Mesmo com estes avanços, a eficácia das medidas de prevenção e, especialmente, de tratamento não melhorou consideravelmente. Sendo assim, as perspectivas das propostas de intervenção tornam-se autolimitadas pelas dúvidas de efetividade oriundas de problemas não satisfatoriamente resolvidos até o início dos anos 90 (Marques, 1992).

Vários fatores limitam a efetividade das medidas propostas, tais como a pobreza do quadro sintomático das anemias, de forma que os pacientes se sentem pouco motivados a tratar de um problema do qual raramente se queixam (Mahomed \& Hytten, 1993; Kanaki, 1994); aparecimento de efeitos colaterais derivados da administração de sais de ferro (Stekel, 1984), produzindo, para o hospedeiro, sintomas mais notáveis que os relatados para a própria anemia, especialmente quando se administram altas doses de sais de ferro; a longa duração do tratamento e, por conseguinte, das queixas resultantes da própria terapia. Uma evidência neste sentido seria o reconhecimento de que o uso de sais de ferro nas doses recomendadas pelas Academais de Pediatria e Obstetrícia re- sulta no aparecimento de efeitos colaterais em $15 \%$ a $20 \%$ dos pacientes ( Cardoso \& Penedo, 1994). O resultado é uma baixa aderência dos usuários aos esquemas recomendados (Stekel, 1984; Kanaki, 1994).

Isto justificaria, portanto, a aparente timidez das metas consensualmente estabelecidas na Reunião de Cúpula de Nova York, em 1990 (UNICEF,1990), bem como a recomendação da Conferência Mundial de Alimentação e Nutrição (OMS, 1992), no sentido de se promoverem novas investigações para se superarem os problemas mais cruciais no enfrentamento das anemais.

\section{Novas perspectivas}

Em animais de experimentação e até em seres humanos já se conhecia, há um bom tempo, o efeito adverso de doses altas e repetidas de ferro, em virtude de mecanismos de bloqueio à absorção, que se tornam progressivamente mais efetivos, reduzindo, em conseqüência, a incorporação do metal ao meio circulante interno (Hanh et al., 1943; Brow et al., 1958).

$O$ efeito bloqueio parece ser cumulativo com o tempo de administração do ferro, de tal forma que uma dose padrão de $100 \mathrm{mg}$ de ferro elementar duas vezes ao dia, em gestantes, apresenta uma absorção de $14 \%$ na primeira semana de tratamento, caindo para $7 \%$ ao final da terceira semana e para apenas $2 \%$ no final da quinta (Halberg, 1992).

Por outro lado, não está bem evidenciada a vantagem de emprego sistemático de polihematínicos, como o uso simultâneo de folato, vitaminas B12 e B6, zinco e ferro, abusivamente promovidos pela indústria farmacêutica e passivamente aprovados pelos profissionais de saúde. É uma forma cara e, na grande maioria dos casos, inútil de se tentar aumentar a eficácia do tratamento.

Num ensaio realizado no Instituto Materno Infantil de Pernambuco (M arques, 1992), demonstrou-se, por exemplo, que altas ou baixas doses de ferro, de forma isolada ou em combinação com folato e B12, não alteram os resultados finais do tratamento. Verificou-se, neste estudo, um aumento progressivo dos casos de reversão, de modo que, aos três meses de tratamento, cerca de $53 \%$ das gestantes tratadas deixaram de ser anêmicas. Dois fatos foram ressaltados: a) a lentidão das respostas terapêuticas, fazendo com que, aos três meses, pouco mais da metade dos casos pudessem ser considerados como curados; b) a constatação de que, mesmo duplicando-se a dose de ferro 
ou acrescentando-se B12 e folato, a expectativa de resposta ao tratamento não melhoraria, ao passo que os custos seriam consideravelmente onerados.

Resultados excepcionalmente favoráveis, com $97 \%$ de cura, foram obti dos recentemente por Suharno et al. (1993), utilizando a combinação ferro +vitamina A, em Java Ocidental, durante oito semanas. São observações, sem dúvida, encorajadoras, não se sabendo, no entanto, se poderiam ser replicadas em outras situações, em razão da reconhecida importância da deficiência de vitamina A na ilha de Java. Todavia, a restauração dos níveis normais de hemoglobina em $68 \%$, ( $\geq 11 \mathrm{mg} / \mathrm{dl}$ em oito semanas, com o uso diário de apenas $60 \mathrm{mg}$ de ferro elementar) pode ser considerado como um resultado altamente positivo (Suharno et al., 1993).

Uma perspectiva um tanto instigante se abre com a proposta de Viteri et al. (1993), com base no princípio de que a mucosa intestinal bloqueia a absorção de ferro medicamentoso quando administrado repetidamente. Desta forma, seria admissível a utilização de doses semanais, uma vez que a mucosa do intestino humano se renova a cada seis dias (Trier, 1968 apud Viteri et al., 1995), ou de esquemas de tratamento duas vezes por semana, em vez de se recomendar a aplicação diária de sais de ferro. Superada assim a barreira bloqueio epitelial, o tratamento seria simplificado, reduzindo custos, efeitos colaterais, aumentando sua eficiência e, por conseguinte, a desejável efetividade

\section{Referências}

ARRUDA, I. K. G., 1990. Prevalência de Anemia em Gestantes de Baixa Renda: Al gumas Variáveis Associadas e sua Repercussão no Récem-Nascido. Tese de Mestrado, Recife: Centro de Ciências da Saúde, Universidade Federal de Pernambuco.

BEHRM AN, E. R. \& KLIEGM AN, R. M., 1994. Nelson Tratado de Pediatria. 14ạ ed., São Paulo: Guanabara Koogan.

BROW, E. B.; DUBAR, C. H. R. \& MOORE, C. V., 1958. Studies in iron transportation and metabolism. XI - Critical analysis of mucosal block of iron in human subjects. Journal of Laboratorial Clinic Medicine, 52:335

CARDOSO, M. A \& PENEDO, M. V. C., 1994. Intervenções nutricionais na anemia. Cadernos de Saúde Pública, 10:231-240.

EBRAHIM, G. J., 1983. Nutritional anaemias. In: Nutrition in Mother and Child Health (G. J. Ebrahim, ed.), pp. 34-53, London: Macmillan. das intervenções em programas de ampla cobertura.

Recentemente, utilizando crianças em um ensaio clínico randomizado e duplo-cego, Schultink et al. (1995), ao aplicarem doses de $30 \mathrm{mg}$ de ferro elementar diariamente e duas vezes semanalmente, ao longo de oito semanas, observaram que não houve diferença estatisticamente significativa entre os dois grupos. Esta posologia de aproximadamente $0,75 \mathrm{mg} /$ $\mathrm{kg} /$ dia de ferro elementar é bastante inferior às recomendadas pelos clássicos tratados de pediatria do Nelson e do Oski, que variam de 4 a 6 $\mathrm{mg} /$ dia de ferro elementar (Behrman, et al., 1994; Martin \& Pearson, 1994).

Um estudo colaborativo já em curso na França e no Senegal, e que conta com a recente adesão do Instituto Materno Infantil de Pernambuco (IMIP), poderá estabelecer condutas inovadoras para o tratamento e, por extensão, para a própria prevenção primária da anemia em gestantes. Admite-se que, dentro dos próximos cinco anos, pode-se esperar um percentual de cura em torno de $90 \%$, o que modificaria consideravelmente o panorama atual, ou seja, a modesta perspectiva de que se possa reduzir em apenas 33\% a prevalência de anemia em mulheres no período reprodutivo. Esta perspectiva representaria um avanço notável, mudando, dentro de uma década, todas as estratégias históricas delineadas em séculos de experiências no controle das anemias nutricionais.
HAHN, P. F.; BALE, W. F. B.; ALFOUR W. M. \&WHIPPLE G. H., 1943. Radioactive iron absorption by the gastrointestinal tract: influence of anemia, anoxia and antecedent feeding: distribuition in growing dogs. Journal of Experimental Medicine, 78:169.

HALBERG, L., 1992. Iron balance in pregnancy and lactation. In: Nutritional Anaemias (S. J. Fomom \& S. Zlotkin, eds.), pp.13-28, Nestlé Nutrition Workshops Series, vol. 30, New York: Vevey/ Raven Press Ltd.

ICNND (Interderpartamental Commitee of Nutrition for National Development), 1965. Northeast Brazil. Nutrition Survey, Washington DC: ICNND. (mimeo.)

INACG (International Nutritional Anemia Consultative Group), 1977. Erradication of Iron Deficiency Anemia. Göteborg: INACG. (mimeo.)

KANAKI, S., 1994. Combate de la anemia en niñas adolescentes: informe de la India. Madres y niños. Boletín sobre Alimentación Infantil y Nutrición Materna, 13:1-3. 
MAHOMED, K. \& HYTEEN, F., 1993. Iron and folate supplementation in pregnancy. In: Effective Care in Pregnancy and Childbirth. (I. Chalmers, M. Enkin \& M. J. N. C. Keirse, eds.), pp. 301-317, New York: Oxford University Press.

MARQUES A. P. O., 1992. Avaliação de Três Esquemas deIntervenção em Gestantes Anêmi cas. Dissertação de Mestrado, Recife: Instituto de Nutrição, Centro de Ciências da Saúde, Universidade Federal de Pernambuco.

MARTIN, P. L. \& PEARSON, H. A., 1994. The anemias. In: Princi ples and Pratice of Pediatrics (F. A. Oski, C. D. DeAngelis, R. D. Feigin, J. A. McMillan \&J. B. Warshaw, eds.), p. 1657-1670, 2nd ed., Philadelphia: Lippincott.

OMS (Organização Mundial de la Salud), 1975. Lucha Contra la Anemia Nutricional, Especialmente Contra la Carencia de Hierro. - Série de Informes Tecnicos, 580. Ginebra: OMS

OMS (Organización Mundial de la Salud), 1992. Informe final de la conferencia. Conferencia Internacional sobre Nutrición. Roma: OMS.

RODRIGUES, O. T. S., 1989. Estudo da Influência de Anemia de Desnutrição Maternas sobre o Peso ao Nascer. Dissertação de Mestrado, São Paulo: Faculdade de Saúde Pública, Universidade de São Paulo.

SALZANO, A. C.; MONTEIRO, E. A.; LAGO, E. E.; FREITAS, L. P. C. G.; LAPA, M. A. G.; BAZANTE, M. O. \& ROMANI, S. A. M., 1975. Pesquisa Nutricional em Três Zonas Fisiográficas do Estado de PernambuCo. Recife: INUFPE/SUDENE. (mimeo.)

SKULTINK, W.; GROSS, R.; GLIWITZKI M.; KARYADI D. \& MATULESSI P., 1995. Effect of daily and twice weekly iron supplementation in Indonesian preschool children with low iron satatus. American Journal of Clinical Nutrition, 61:111-115.
STEKEL, A., 1984. Prevention of iron deficiency. In: Iron Nutrition in Infancy and Childhood (A. Stekel, eds.), pp. 179-194, Nestlé Nutrition Workshop Series, vol. 4, New York: Vervey/ Raven Press.

SUHARNO, D.; WEST, C. E.; MUHILAI; KARYADI, D. \& HAUTVAST, J. G. A., 1993. Suplementation with vitamin $A$ and iron for nutritional anaemia in pregnant women in West Java, Indonesia. The Lancet, 342:1325-1328.

SZARFARC, S. C., 1972. Anemia Ferropriva em Parturientes e Recém-Nasci dos de um Grupo Populacional de Baixo Nível Sócio-Econômico de São Paulo. Tese de Doutorado, São Paulo: Faculdade de Saúde Pública, Universidade de São Paulo.

SZARFARC, S. C., 1983. Prevalência de Anemia Nutricional entre Gestantes Atendidas em Centros de Saúde do Estado de São Paulo. Tese de Livre Docência, São Paulo: Faculdade de Saúde Pública, Universidade de São Paulo.

TORRES, M. A. A.; SATO, K.; JULIANO Y. \& QUEIROZ, S. S., 1993. O leite fortificado, como medida de intervenção no combate a anemia carencial ferropriva, em crianças atendidas em Unidade Básica de Saúde (UBS). XV International Congress of Nutrition. Adelaide, Austrália.

UNICEF (Fundo das Nações Unidas para a Infância), 1990. Estratégia para Melhorar a Nutrição de Crianças e Mulheres nos Países em Desenvolvimento. New York: UNICEF

VITERI, F. E.; HERCBERG, S.; GALAN, P.; GUIRO, A. \& PREZIOSI, P., 1993. Absorption of iron supplements administered daily or weekly: a collaborative study. NestléFoundation, Annual Report, 83-96. 